\title{
SUFFICIENT CONDITIONS FOR PERIODICITY OF A KILLING VECTOR FIELD
}

\author{
WALTER C. LYNGE
}

\begin{abstract}
Let $X$ be a complete Killing vector field on an $n$ dimensional connected Riemannian manifold. Our main purpose is to show that if $X$ has as few as $n$ closed orbits which are located properly with respect to each other, then $X$ must have periodic flow. Together with a known result, this implies that periodicity of the flow characterizes those complete vector fields having all orbits closed which can be Killing with respect to some Riemannian metric on a connected manifold $M$. We give a generalization of this characterization which applies to arbitrary complete vector fields on $M$.
\end{abstract}

THEOREM. Let $X$ be a complete Killing vector field on a connected, $n$ dimensional Riemannian manifold $M$. Assume there are $n$ distinct points $p$, $p_{1}, \cdots, p_{n-1}$ in $M$ such that the respective orbits $\gamma, \gamma_{1}, \cdots, \gamma_{n-1}$ of $X$ through them are closed and $\gamma$ is nontrivial. Suppose further that each $p_{i}$ is joined to $p$ by a unique minimizing geodesic and $d\left(p, p_{i}\right)=\eta_{i}<D / 2$, where d denotes distance on $M$ and $D$ is the diameter of $\gamma$ as a subset of $M$. Let $w_{1}, \cdots, w_{n-1}$ be the unit vectors in $T_{p} M$ such that $\exp \eta_{i} w_{i}=p_{i}, i=1$, $\cdots, n-1$. Assume that the vectors $X_{p}, w_{1}, \cdots, w_{n-1}$ span $T_{p} M$. Then the flow $\varphi_{t}$ of $X$ is periodic.

Proof. Fix $i$ in $\{1,2, \cdots, n-1\}$. We first show that the orbit $\gamma_{i}$ does not lie entirely in the sphere $S_{p}\left(\eta_{i}\right)$ of radius $\eta_{i}$ about $p$. For suppose it did. Let $q$ be a point in $\gamma$ such that $d(p, q)=D$. Let $s$ be a real number such that $\varphi_{s}(p)=q$. Then $d\left(p, \varphi_{s}\left(p_{i}\right)\right)=\eta_{i}$ by our supposition. Since $\varphi_{s}$ is an isometry, $d\left(q, \varphi_{s}\left(p_{i}\right)\right)=\eta_{i}$. By the triangle inequality, $d(p, q) \leqq d\left(p, \varphi_{s}\left(p_{i}\right)\right)+$ $d\left(\varphi_{s}\left(p_{i}\right), q\right)$. Thus $D \leqq 2 \eta_{i}$. This contradicts the hypothesis of the theorem.

Let $\lambda$ and $\lambda_{i}$ denote the prime periods of $\gamma$ and $\gamma_{i}$ respectively, assuming for the moment that $\gamma_{i}$ is nontrivial. We claim that $\lambda_{i} / \lambda$ is rational. Suppose it is not. Let $s$ be any number in the interval $\left(0, \lambda_{i}\right)$. We recall that it is possible to find integers $m$ and $n$ such that $\left|m \lambda-n \lambda_{i}-s\right|$ is as small as we please. Furthermore, since $\gamma_{i}$ is closed, the map from the reals to $M$ given by $t \rightarrow \varphi_{t}\left(p_{i}\right)$ is uniformly continuous. Let $\varepsilon>0$ be given. Then

Received by the editors June 28, 1972 .

AMS (MOS) subject classifications (1970). Primary 53C20, 53B20; Secondary 57D25.

Key words and phrases. Killing vector field, isometry, flow, Lie algebra, Riemannian metric, Riemannian manifold, closed orbit, minimizing geodesic.

(c) American Mathematical Society 1973 
there exists $\delta>0$ such that $d\left(\varphi_{m \lambda}\left(p_{i}\right), \varphi_{n \lambda_{i}+t}\left(p_{i}\right)\right)<\varepsilon$ whenever

$$
\left|m \lambda-\left(n \lambda_{i}+t\right)\right|<\delta \text {. }
$$

Now given any $s$ in $\left(0, \lambda_{i}\right)$, pick $m$ and $n$ so that $\left|m \lambda-\left(n \lambda_{i}+s\right)\right|<\delta$. Then $d\left(\varphi_{m \lambda}\left(p_{i}\right), \varphi_{n \lambda_{i}+s}\left(p_{i}\right)\right)<\varepsilon$. But $\varphi_{n \lambda_{i}+s}\left(p_{i}\right)=\varphi_{s}\left(p_{i}\right)$, since $n \lambda_{i}$ is a multiple of the period of $\gamma_{i}$. Thus $d\left(\varphi_{m \lambda}\left(p_{i}\right), \varphi_{s}\left(p_{i}\right)\right)<\varepsilon$. Moreover, $\varphi_{m \lambda}\left(p_{i}\right)$ lies in $S_{p}\left(\eta_{i}\right)$, since $d\left(p, \varphi_{m \lambda}\left(p_{i}\right)\right)=d\left(\varphi_{m \lambda}(p), \varphi_{m \lambda}\left(p_{i}\right)\right)=d\left(p, p_{i}\right)=\eta_{i}$. We have shown that given any point $\varphi_{s}\left(p_{i}\right)$ in $\gamma_{i}$ and given any $\varepsilon>0$, there exists a point in $S_{p}\left(\eta_{i}\right)$, namely $\varphi_{m \lambda}\left(p_{i}\right)$, such that $d\left(\varphi_{m \lambda}\left(p_{i}\right), \varphi_{s}\left(p_{i}\right)\right)<\varepsilon$. Thus $\gamma_{i} \subset S_{p}\left(\eta_{i}\right)$, contradicting what we proved in the previous paragraph. Hence $\lambda_{i} / \lambda$ is rational.

We write $n_{i} \lambda_{i}=m_{i} \lambda$ for integers $n_{i}, m_{i}$. We have $p_{i}=\varphi_{n_{i} \lambda_{i}}\left(p_{i}\right)=\varphi_{m_{i} \lambda}\left(p_{i}\right)$. Let $N$ be the least common multiple of the $m_{i}$ as $i$ ranges through the indices for which $\gamma_{i}$ is nontrivial. If all the $\gamma_{i}$ are trivial, take $N$ to be 1 . Then we have $\varphi_{\lambda N}\left(p_{i}\right)=p_{i}, i=1, \cdots, n-1$. Since also $\varphi_{\lambda, V}(p)=p$, we have that every point of the unique minimizing geodesic from $p$ to $p_{i}$ is left fixed by $\varphi_{\lambda N^{\prime}}$. Thus $\varphi_{\lambda N^{*}}(p) w_{i}=w_{i}, i=1, \cdots, n-1$. Since also $\varphi_{\lambda N^{*}}(p) X_{p}=$ $X_{p}$, we have that $\varphi_{\lambda N^{*}}(p)$ is the identity on $T_{p} M$. Hence $\varphi_{\lambda N}$ is the identity on $M\left[1\right.$, p. 62]. Hence the flow $\varphi_{t}$ of $X$ is periodic.

A special case of this theorem is: Assuming $X$ is nontrivial, the flow of $X$ is periodic if there is an open set $U$ such that all the orbits of $X$ which meet $U$ are closed. We remark that this special case can be proved from a different and more extrinsic point of view than the theorem itself. Let $H$ be the closure in the isometry group $G$ of $M$ of the 1-parameter subgroup of $G$ corresponding to $X$. Then $H \approx T^{m}$ for some $m \geqq 1$. By considering the action of $H$ on $M$, it can be shown that $m=1$. We omit the details.

It is known that if $X$ is a complete vector field having periodic flow on a connected manifold $M$, then there is a Riemannian metric $g$ on $M$ such that $X$ is Killing with respect to $g$. From this and the theorem, it follows that periodicity of the flow characterizes those complete vector fields having all orbits closed which can be Killing with respect to some Riemannian metric on $M$. In conclusion, we note that this characterization can easily be generalized to apply to arbitrary complete vector fields on $M$ as follows:

Proposition. Let $X$ be a complete vector field on a connected manifold $M$. Then there exists a Riemannian metric $g$ on $M$ such that $X$ is Killing with respect to $g$ if and only if there exist (for some positive integer $m$ ) complete vector fields $X_{1}, \cdots, X_{m}$ on $M$ such that

(i) The flows of the $X_{i}$ are periodic;

(ii) $\left[X_{i}, X_{j}\right]=0, i, j=1, \cdots, m$;

(iii) $X=\sum_{i=1}^{m} c_{i} X_{i}, c_{i}$ real numbers. 
Proof. Suppose $X$ is Killing with respect to a metric $g$. Let $G$ be the isometry group of $(M, g)$. We recall that the Lie algebra $g$ of $G$ is naturally isomorphic with the Lie algebra of complete Killing vector fields on $M$. Given $Y$ in $\mathfrak{g}$, we denote by $Y^{*}$ the corresponding complete Killing vector field. Now let $A$ in $\mathfrak{g}$ be such that $A^{*}=X$. If $\exp t A$ is a closed 1-parameter subgroup of $G$, then the flow of $X$ is periodic and we are finished. If $\exp t A$ is not closed, let $H$ be its closure in $G$. Then $H$ is isomorphic to some toral group $T^{m}$. Let $\left\{A_{1}, \cdots, A_{m}\right\}$ be a basis for the Lie algebra of $H$ such that $\exp t A_{i}, i=1, \cdots, m$, are closed 1-parameter subgroups of $H$. Then $A=\sum_{i=1}^{m} d_{i} A_{i}$ for real numbers $d_{i}$. Set $X_{i}=A_{i}^{*}$ and $c_{i}=d_{i}, i=1, \cdots, m$. (i), (ii), and (iii) are obviously satisfied.

To prove the sufficiency, let $\varphi^{i}$ be the flow of $X_{i}$, and let $\lambda_{i}$ denote the period of $\varphi^{i}$. Let $\tilde{g}$ be any Riemannian metric on $M$ and define a new metric $g$ by

$$
g(u, v)=\int_{0}^{\lambda_{m}} \int_{0}^{\lambda_{m-1}} \cdots \int_{0}^{\lambda_{1}} \tilde{g}_{\varphi_{t}(p)}\left(\varphi_{t^{*}}(p) u, \varphi_{t^{*}}(p) v\right) d t^{1} d t^{2} \cdots d t^{m}
$$

where $p$ is a point of $M, u, v$ are vectors in $T_{p} M$, and $\varphi_{t}=\varphi_{t^{1}}^{1} \circ \cdots \circ \varphi_{t^{m}}^{m}$. Since $\left[X_{i}, X_{j}\right]=0, i, j=1, \cdots, m$, the flows $\varphi^{i}$ commute with each other. From this it follows that each $\varphi^{i}$ is an isometric flow with respect to $g$, and hence each $X_{i}$ is Killing with respect to $g$. Thus $X$ is also Killing with respect to $g$.

\section{BIBLIOGRAPHY}

1. Sigurdur Helgason, Differential geometry and symmetric spaces, Pure and Appl. Math., vol. 12, Academic Press, New York, 1962. MR 26 \#2986.

Department of Mathematics, Edgecliff College, Cincinnati, Ohio 45206

Current address (Walter C. Lynge): 6501 N. Brookwood Lane, Peoria, Illinois 61614 